- Divulgare ai non professionisti della salute, utilizzando un linguaggio adatto a tutti ma un profondo rigore scientifico, le principali novità riguardanti la psicologia e la psicoterapia

- Formare i professionisti della salute attivi sul territorio ticinese agli aspetti inerenti la nostra attività di ricerca

Campi di applicazione principali per «Psychology Research Ticino»:

- Valutazione e promozione della salute mentale e del benessere psicologico nella popolazione residente in Canton Ticino
- Studio e prevenzione dei comportamenti a rischio negli adolescenti e giovani adulti residenti in Canton Ticino

- Valutazione delle problematiche e delle difficoltà di adattamento negli adolescenti e nei giovani adulti residenti in Canton Ticino

- Valutazione delle problematiche e delle difficoltà di adattamento delle famiglie residenti in Canton Ticino

Link del sito di «Psychology Research Ticino»: https://www.psychologyresearchticino.ch/Home

Nicola Gianinazzi è membro di comitato e delegato per la Svizzera italiana.

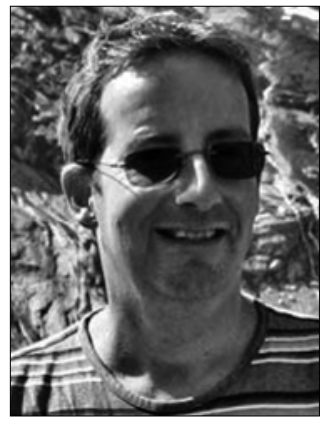

\title{
Nouvelles de la Suisse italienne
}

Nicola Gianinazzi

À l'occasion du 40e anniversaire de l'ASP, nous publions ce petit paragraphe historique.

Dans la dernière décennie, l'ASP a vu naître sa section Suisse italienne, née de l'initiative de son directeur de l'époque, Emil Stutz, et de moi-même, dans le but de redynamiser le versant italophone de l'Association. Dès la première réunion de "constitution ", en 2010, la participation fut forte avec une vingtaine de membres (soit la quasi-totalité des adhérents de langue italienne) et le dîner sympathique qui s'ensuivit marqua définitivement le lancement officiel de l'expérience.

La Section a amené beaucoup de nouveaux membres à l'ASP - un certain nombre de jeunes notamment, mais aussi de moins jeunes - un membre collectif et, récemment, un de ses délégués siège à la Commission consultative cantonale des professions de la psychologie et de la psychothérapie.

Aujourd'hui, ses activités de conseil, de mise en réseau, dorganisation de formations et de médiation culturelle sont mises en avant depuis plus d'un an sous la forme plus concise d'une délégation Suisse italienne.

\section{Depuis la Suisse italienne}

Nous fournissons régulièrement des conseils personnalisés à des confrères psychologues et psychothérapeutes et, occasionnellement, à des instituts de formation suisses et étrangers et à d'autres institutions privées et publiques.

Par ailleurs, au sein de l'association, le travail consiste surtout en une médiation entre les entités régionale et nationale, en conseils, en révisions et en traductions de textes d'information ou à caractère technique.

Nous poursuivons notre collaboration fructueuse avec l'Istituto Ricerche di Gruppo (IRG) pour lorganisation de la formation continue, de manière à pouvoir garantir le maintien d'un vaste éventail de cours de qualité. Les cours peuvent ainsi être choisis dans le cadre du parcours de formation postgrade selon le concept ASP Integral.

En outre, depuis 2018, je suis entré dans la rédaction de Sciences psychothérapeutiques, la Suisse italienne et le contexte italien vont ainsi gagner en présence et en visibilité dans notre revue thématique.

Les contacts avec des professeurs de différentes universités et facultés du Tessin se poursuivent. Cette activité stimulante de relations publiques est utile et nécessaire pour enrichir les propositions de formation et les contributions aux revues de l'association. Dans ce cadre, la collaboration rédactionnelle avec la revue Psicoterapia e scienze umane, codirigée par Paolo Migone, est également renforcée. 
Panorama des psychothérapies dans le Tessin et au-delà des frontières

Dans le Tessin - dans le contexte d'une collaboration internationale impliquant plusieurs membres de l'ASP, des chercheurs universitaires et d'autres partenaires - un site consacré à la recherche dans le domaine de la santé et de la médecine, de la psychothérapie, de la psychologie et des sciences humaines en général a été créé. Sa mission consiste à identifier, concevoir et mettre en œuvre des projets de recherche qui explorent des thématiques psychologiques et psychosociales importantes pour les citoyens résidents dans le canton du Tessin.

Nos projets nont pas exclusivement une finalité de connaissance, même si, en tant que chercheurs, nous accordons une valeur fondamentale au savoir. Ils visent à approfondir les thèmes et phénomènes psychologiques et psychosociaux particulièrement importants pour les résidents du Tessin. En effet, lors du développement de chacun de nos projets, nous portons une attention particulière aux particularités, aux spécificités et avant tout aux exigences de notre territoire. La Mission de « Psychology Research Ticino » ne se résume pas à l'observation et à la classification des phénomènes psychologiques et sociaux qui se développent dans le Tessin: nous voulons utiliser les connaissances issues de nos études pour proposer et construire de programmes déducation, de promotion et de soutien à la santé qui puissent réellement être mis en œuvre sur le territoire et qui soient utiles et fonctionnels pour améliorer la qualité de vie des résidents de notre canton. Voici en quelques mots la mission de «Psychology Research Ticino » :

- Promouvoir la recherche scientifique dans les domaines psychologique et psychosocial sur le territoire tessinois

- Impliquer activement les résidents du canton dans les projets de recherche développés par « Psychology Research Ticino » en favorisant un climat de collaboration, de participation active et de responsabilité individuelle à légard des sujets liés à la santé

- Diffuser auprès des résidents du canton les résultats des projets de recherche développés par " Psychology Research Ticino " dans le but d'" éduquer » les citoyens sur des sujets liés à la santé et au bien-être, essentiels à la qualité de vie des individus
- Divulguer les principales actualités dans les domaines de la psychologie et de la psychothérapie auprès de personnes extérieures aux professions de la santé, en utilisant un langage adapté à tous tout en gardant une grande rigueur scientifique

- Former les professionnels de la santé actifs sur le territoire tessinois aux aspects inhérents à notre activité de recherche

Principaux champs d'application de « Psychology Research Ticino »:

- Évaluation et promotion de la santé mentale et du bien-être psychologique dans la population résidant dans le canton du Tessin

- Étude et prévention des comportements à risque chez les adolescents et les jeunes adultes résidant dans le Tessin

- Évaluation des problématiques et des difficultés d'adaptation chez les adolescents et les jeunes adultes résidant dans le Tessin

- Évaluation des problématiques et des difficultés d’adaptation des familles résidant dans le Tessin

Lien du site de « Psychology Research Ticino »: https://www.psychologyresearchticino.ch/Home

Nicola Gianinazzi est membre de comité et délégué pour la Suisse italienne.

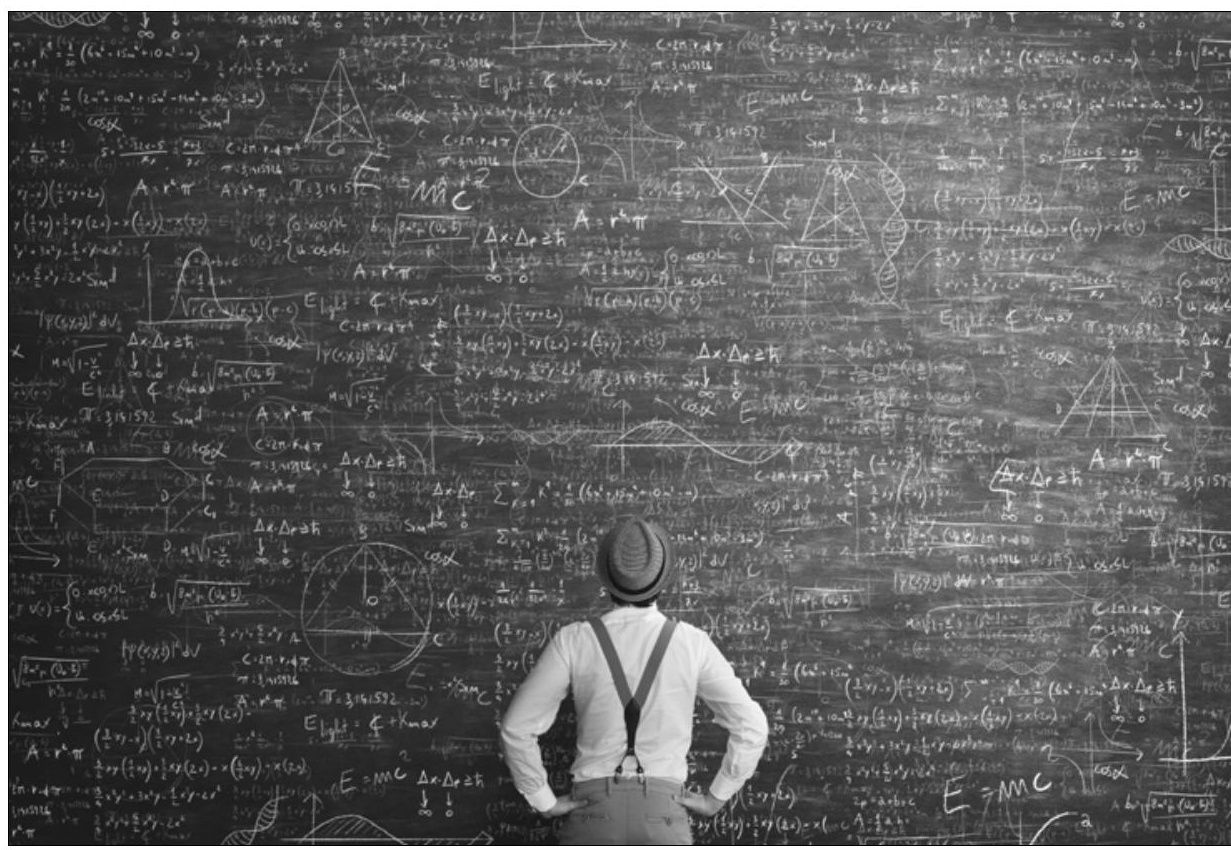

